\title{
Maternal engineered nanomaterial inhalation during gestation alters the fetal transcriptome
}

\author{
P. A. Stapleton ${ }^{1,2 \dagger}$, Q. A. Hathaway ${ }^{3,4,6 \dagger}$, C. E. Nichols ${ }^{5}$, A. B. Abukabda $a^{6,7}$, M. V. Pinti ${ }^{3,4}$, D. L. Shepherd ${ }^{3,4}$, \\ C. R. McBride ${ }^{6,8}$, J. Yi, ${ }^{6,8}$, V. C. Castranova ${ }^{6,7}$, J. M. Hollander ${ }^{3,4,6}$ and T. R. Nurkiewicz ${ }^{4,6,8^{*}}$
}

\begin{abstract}
Background: The integration of engineered nanomaterials (ENM) is well-established and widespread in clinical, commercial, and domestic applications. Cardiovascular dysfunctions have been reported in adult populations after exposure to a variety of ENM. As the diversity of these exposures continues to increase, the fetal ramifications of maternal exposures have yet to be determined. We, and others, have explored the consequences of ENM inhalation during gestation and identified many cardiovascular and metabolic outcomes in the F1 generation. The purpose of these studies was to identify genetic alterations in the F1 generation of Sprague-Dawley rats that result from maternal ENM inhalation during gestation. Pregnant dams were exposed to nano-titanium dioxide (nano- $\left.\mathrm{TiO}_{2}\right)$ aerosols $(10 \pm 0$. $5 \mathrm{mg} / \mathrm{m}^{3}$ ) for 7-8 days (calculated, cumulative lung deposition $=217 \pm 1 \mu \mathrm{g}$ ) and on GD (gestational day) 20 fetal hearts were isolated. DNA was extracted and immunoprecipitated with modified chromatin marks histone 3 lysine 4 tri-methylation (H3K4me3) and histone 3 lysine 27 tri-methylation (H3K27me3). Following chromatin immunoprecipitation (ChIP), DNA fragments were sequenced. RNA from fetal hearts was purified and prepared for RNA sequencing and transcriptomic analysis. Ingenuity Pathway Analysis (IPA) was then used to identify pathways most modified by gestational ENM exposure.
\end{abstract}

Results: The results of the sequencing experiments provide initial evidence that significant epigenetic and transcriptomic changes occur in the cardiac tissue of maternal nano- $\mathrm{TiO}_{2}$ exposed progeny. The most notable alterations in major biologic systems included immune adaptation and organismal growth. Changes in normal physiology were linked with other tissues, including liver and kidneys.

Conclusions: These results are the first evidence that maternal ENM inhalation impacts the fetal epigenome.

Keywords: Nanotechnology, Toxicology, Nanomaterial, Inhalation, Epigenetics, Maternal, Fetal

\section{Background}

The Barker Hypothesis [1], Developmental Origins of Health and Disease (DOHaD) [2], and fetal programming [3], all explore the relationship between the health of the gestational environment and fetal development and how this predisposes to future disease or sensitivities. Maternal prenatal health challenges such as

\footnotetext{
* Correspondence: tnurkiewicz@hsc.wvu.edu

${ }^{\dagger}$ Equal contributors

${ }^{4}$ Mitochondria, Metabolism \& Bioenergetics Working Group, West Virginia University School of Medicine, Morgantown, WV, USA

${ }^{6}$ Toxicology Working Group, West Virginia University School of Medicine, Morgantown, WV, USA

Full list of author information is available at the end of the article
}

nutrient deficiency, undernourishment, gestational diabetes and hypertension have been linked to an elevated risk for postnatal cardiovascular diseases [4]. Recently, maternal environmental toxicant exposures have become of prominent interest in relation to the impact of exposure on the fetal milieu and subsequent progeny health [5]. We have reported that maternal ENM inhalation impairs the ability of uterine arterioles to properly dilate, and this impacts litter health in the form of pup weight, number and gender distribution; as well as impaired microvascular function [21]. While these studies have focused on the maternal development of a hostile gestational environment and subsequent reduction in fetal 
nutrients, fetal epigenetic modifications may also occur. Conceptually, this relationship is not novel, but applications of environmental toxicants to the maternal-fetal models are. For example, bisphenol A [6] and air pollution [7] have been shown to negatively impact fetal outcomes. However, the impact of maternal ENM on fetal health and/or epigenetic modification are poorly understood.

Despite the ubiquitous inclusion of engineered nanomaterials in widespread applications, and their projected proliferation in human endeavors, the consequences of maternal ENM inhalation on the developing fetus and their impacts on future health are at best, vague, yet they are increasingly becoming a health concern. The prevalence of ENM covers an immense spectrum: surface coatings and additives in common consumer products (electronics, food, cosmetics), additives in industrial processes (advanced building materials, synthetic fuels), and components of clinical applications (diagnostics, drug delivery, implantable devices). It is widely recognized that throughout the ENM life cycle, the greatest risk for human exposure and subsequent health consequences begins with ENM inhalation, and is typically followed by systemic injuries. We have reported that pulmonary and systemic microvascular inflammation [29, 32] follow ENM inhalation exposure. Consistent with this, other systemic morbidities known to follow pulmonary ENM exposures include: inflammation/apoptosis $[8,9]$, macrovascular and microvascular dysfunction [10], atherogenesis [11], and organ level ischemia [12]. The developing fetus is equally a systemic target of numerous anthropogenic toxicants [13].

The impact of gestational ENM exposures on maternal and fetal health have been increasingly studied in the past decade. Adverse impact of ENM exposures on maternal health [14] and pregnancy $[15,16]$ have been reported in animal models. Teratogenic and embryo-lethal effects associated with ENM exposure have been shown [17]. The outcomes from several studies also highlight post-natal behavioral deficits $[18,19]$, cardiovascular $[20$, 21], renal [15], immune [22], reproductive [23, 24], pulmonary, and metabolic $[20,25]$ abnormalities.

Epigenetics, or the transient control of genes through DNA methylation or histone modification, is a recent area of intense focus by governmental agencies recognizing mechanistic links between environmental toxicants and gene expression [26]. These adverse maternal and fetal outcomes strongly reflect the potential risk of ENM exposure during pregnancy that may be linked. However, given the inherent physiological dependencies and complexities of developing and maintaining a healthy pregnancy, linking the mechanisms of pulmonary exposure and gestational effects remains very challenging. Given the magnitude of and the complexity these transgenerational effects, the most effective approach may be to initiate studies from the fetal epigenome and/or transcriptome. This is largely because fetal epigenetic outcomes resulting from maternal ENM exposure consequences may be caused by the creation of a hostile gestational environment [27], and/or the direct impact of ENM interacting with the developing embryo [13]. Because either of these possibilities would compromise health, the purpose of these studies was to identify epigenetic changes in cardiac gene expression within the maternally exposed F1 generations. We hypothesized that because maternal ENM inhalation lead to uterine microvascular dysfunction [21], this contributes to a hostile gestational environment, and altered fetal gene expression results. To test this, pregnant dams were intermittently exposed to nano- $\mathrm{TiO}_{2}$ aerosols during gestational days 5-19, and their litters were studied on GD 20.

\section{Methods}

\section{Animal model}

Sprague Dawley rats were purchased from Hilltop Laboratories (250-275 g female; 300-325 g male). All experiments were approved by the West Virginia University Animal Care and Use Committee and experiments adhered to the National Institutes of Health (NIH) Guide for the Care and Use of Laboratory Animals (8th Ed.). Rats were provided food and water ad libitum and housed in an AAALAC approved animal facility at the West Virginia University Health Sciences Center. Before mating, rats were acclimated for a minimum of $72 \mathrm{~h}$, as previously described [20]. Pregnancy was verified by identification of the vaginal plug, after which, rats were randomly placed into one of two nano$\mathrm{TiO}_{2}$ exposure groups. These two exposure groups were virtually identical and were created to generate a discrete tissue bank for RNA sequencing, or ChIP sequencing.

\section{Engineered Nanomaterial}

Nano- $\mathrm{TiO}_{2}$ P25 powder was purchased from Evonik (Aeroxide TiO2, Parsippany, NJ), containing anatase $(80 \%)$ and rutile $(20 \%) \mathrm{TiO}_{2}$. Nano- $\mathrm{TiO}_{2}$ was prepared by drying, sieving, and storing, as previously described $[28,29]$. Nano- $\mathrm{TiO}_{2}$ aerosols were created with our aerosol generator (US Patent No. 8,881,997) [30]. Particle characteristics have been determined including the primary particle size $(21 \mathrm{~nm})$, the specific surface area $\left(48.08 \mathrm{~m}^{2} / \mathrm{g}\right)[29,31]$, and the Zeta potential $(-56.6 \mathrm{mV})$ [32].

\section{Nano- $\mathrm{TiO}_{2}$ inhalation exposures}

The nano-particle aerosol generator (US Patent No. $8,881,997)$ and whole-body inhalation exposure system used for the current study have been described extensively in previous studies $[29,31]$. This collective exposure system consists of a vibrating fluidized bed, a Venturi vacuum pump, cyclone separator, impactor and mixing device, an 
animal housing chamber, and real-time monitoring devices with feedback control. Nano- $\mathrm{TiO}_{2}$ was aerosolized via a high velocity air stream passing through the vibrating fluidized bed and into the Venturi vacuum pump. The generated aerosols then entered the cyclone separated, which is designed to remove agglomerates $>400 \mathrm{~nm}$ at an input flow rate of $60 \mathrm{l} / \mathrm{min}$ of clean dry air before entering the exposure chamber.

Size distribution, mean aerodynamic diameter, and relative mass concentration of the aerosols were monitored in real time (Electrical Low Pressure Impactor (ELPI), Dekati, Tempere, Finland) while the particle size distribution was also measured in real-time with a Scanning Mobility Particle Sizer device (SMPS; TSI Inc., St. Paul, MN). These measurements were verified throughout a given exposure by collecting nanoparticle samples on filters, and making hourly gravimetric measurements with a microbalance. This approach was also used to collect samples for transmission electron microscopy.

Inhalation exposures were initiated on GD $5.78 \pm 0.11$ and lasted for $7.79 \pm 0.26$ days of gestation. Exposure days were not consecutive to decrease animal stress. Once the steady state nano- $\mathrm{TiO}_{2}$ aerosol concentration was achieved, exposure duration was adjusted to produce a daily calculated lung deposition of $31 \pm 1.1 \mu \mathrm{g}$ per day, and the cumulative, calculated dose was therefore $217 \pm 1.0 \mu \mathrm{g}$. Lung deposition was calculated based on previously described mouse methodology, and normalized to rat weight and to pregnant rat minute ventilation using the equation: $\mathrm{D}=\mathrm{F} \cdot \mathrm{V} \cdot \mathrm{C} \cdot \mathrm{T}$, where $\mathrm{F}$ is the deposition fraction (14\%), $\mathrm{V}$ is the minute ventilation based on body weight, $C$ equals the mass concentration $\left(\mathrm{mg} / \mathrm{m}^{3}\right)$, and $\mathrm{T}$ equals the exposure duration (minutes) $[29,33]$. The target concentration was $10 \mathrm{mg} / \mathrm{m}^{3}$ and the duration was 4-6 $\mathrm{h} /$ exposure (depending on the steady state concentration, as this was used to calculate the lung burden). The last exposure was conducted $24 \mathrm{~h}$ prior to sacrifice and experimentation. Control animals were exposed to HEPA filtered air only.

\section{Chromatin Immunoprecipitation (ChIP) sequencing Isolation}

Cardiac tissue was isolated from GD 20 pups in both the nano- $\mathrm{TiO}_{2}$ exposure and control groups. Each litter was considered an $n=1$, with cardiac tissue from 5 to 6 pups within each litter being pooled together to collect enough tissue ( $25 \mathrm{mg})$. Chromatin Immunoprecipitation (ChIP) was carried out using the MAGnify ${ }^{\mathrm{Tm}}$ Chromatin Immunoprecipitation System (Thermo Fisher, Rockford, IL) per manufacturer's instructions. Briefly, hearts were homogenized and treated with $37 \%$ formaldehyde, which was prepared fresh. Cross-linking was stopped with $1.25 \mathrm{M}$ glycine. Samples were pelleted through centrifugation and washed in D-PBS before sonication. Using a Sonicator
Ultrasonic Processor XL2015 (Misonix Sonicator, Farmingdale, NY) chromatin was sheared to a size of 500-700 base pairs, determined using gel electrophoresis (Fig. 1a). Chromatin was then isolated through ultracentrifugation $(20,000 \mathrm{~g})$ and diluted to $\sim 60 \mathrm{uL}$ of chromatin per immunoprecipitation reaction. Samples from both the control and nano- $\mathrm{TiO}_{2}$ cohorts were incubated with histone $3 \mathrm{ly-}$ sine 4 tri-methylation (H3K4me3, product number: G.532.8, Thermo Fisher, Rockford, IL) or histone 3 lysine 27 tri-methylation (H3K27me3, product number: G.299.10, Thermo Fisher, Rockford, IL) antibody bound beads. These are two of the most prominently studied and classically applied for activation/repression analysis of gene activity. After incubation, samples were treated to reverse cross-linking solution and Proteinase $K$ to remove bound proteins. DNA was then eluted from beads, using heat, and quantified using a Qubit (Thermo Fisher, Rockford, IL). The TruSeq ChIP Library Preparation Kit (Illumina, Inc., San Diego, CA) was implemented to build the libraries.

\section{ChIP bioinformatics}

Samples were processed using the Illumina MiSeq (Illumina, Inc., San Diego, CA) at the West Virginia University Genomics Core, ran as paired-end reads. Fastq files were assessed for quality using FastQC (Babraham Bioinformatics) (Fig. 1b), where it was determined that partial trimming was needed. Trimming of fastq files was accomplished through Trimmomatic [34] (Fig. 1c). Reads were then mapped to the rat genome (rn6) using the default parameters in bowtie2. To perform differential binding analysis on reads while distinguishing peaks, diffReps was used [35]. Bedtools functions were used to delineate upstream promoter regions of genes (bedtools slop) and evaluate the promoter/gene overlay (bedtools intersect). Genes were defined to include 1000 bases upstream from the start of the gene, indicative of our selected "promoter region."

\section{RNA sequencing Isolation}

Cardiac tissue was procured through the same methods as listed above in the ChIP Sequencing section. RNA was then isolated from heart tissue using the Vantage ${ }^{\text {тм }}$ Total RNA Purification Kit (Origene, Rockville, MD) per manufacturer's instructions. Briefly, tissue was homogenized and lysis buffer was added to the sample. Sample RNA was spin-column purified and measured for RNA concentration using the Qubit (Thermo Fisher, Rockford, IL). Library preparation was performed using TruSeq RNA Library Prep Kit v2 (Illumina, Inc., San Diego, CA). Quality of RNA was determined using the Agilent 2100 BioAnalyzer (Agilent Technologies, Santa Clara, CA); degradation of cytosolic ribosomal RNAs (28S and 


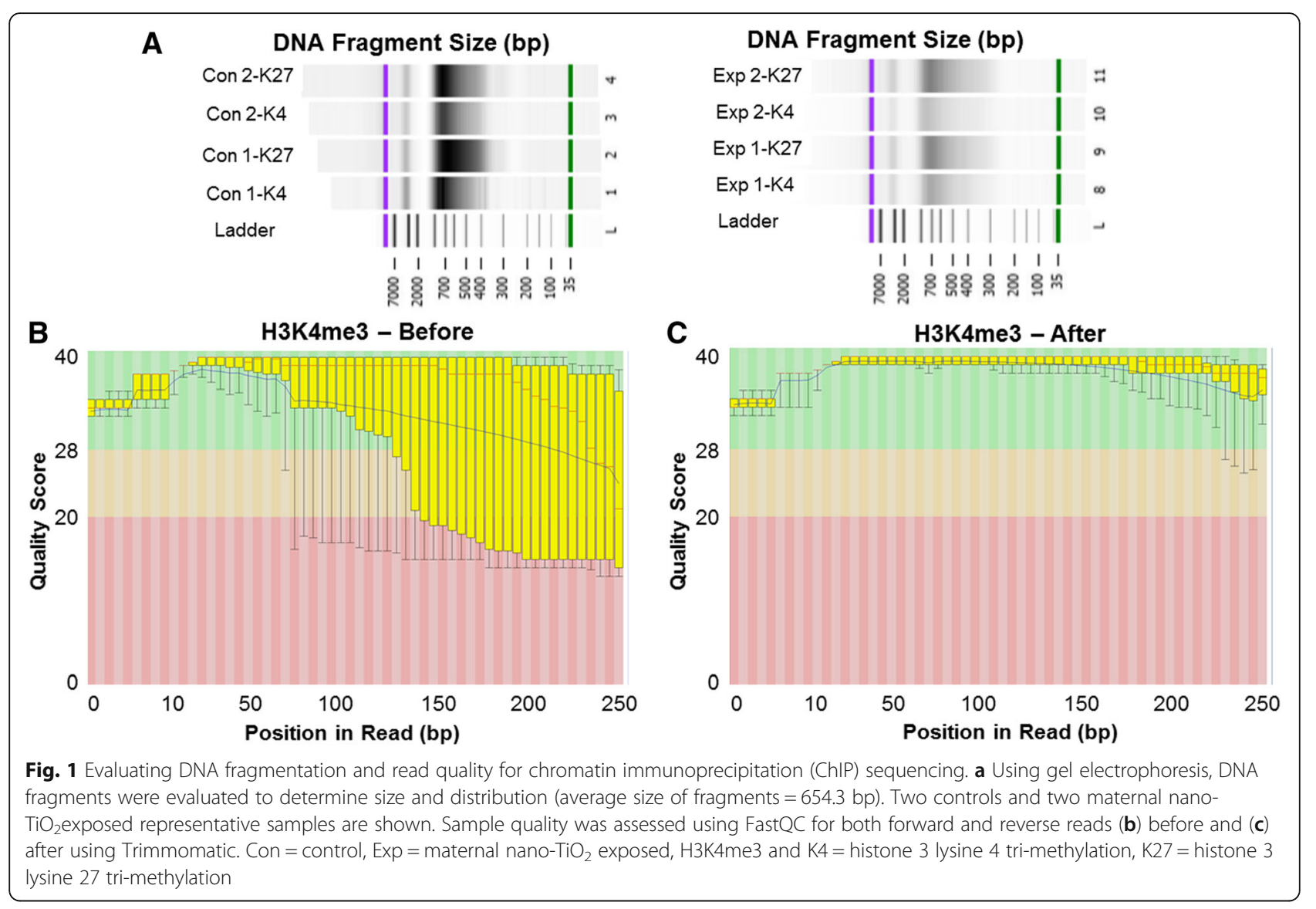

18S) are used as a measure of the total RNA Integrity Number (RIN) (Fig. 2a, b).

\section{RNA bioinformatics}

Samples were processed using the Illumina HiSeq (illumina, Inc., San Diego, CA) at Marshall University. Samples were run as paired-end reads. Paired-end, fastq files were aligned with HISAT2 [36] to the rat genome (rn6) without trimming. Samtools 1.2 [37] was used for the conversion of SAM to BAM format. Counts data was prepared using Subread 1.5.2 [38], specifically featureCounts [39]. Differential expression analysis was accomplished using DESeq2 [40] in R.

\section{Ingenuity pathway analysis (IPA)}

Protein ontology and pathway analysis were completed through QIAGEN's IPA (www.qiagen.com/ingenuity) software. Core analyses and comparative analyses were run on individual and combined ChIP and RNA data sets, respectively. Z-scores are representative of fold change between groups.

RNA IPA Protein Ontology.

The intensity of the color, moving toward blue or red, indicates the degree to which a specific pathway is being decreased or increased, respectively. The change in color, reflective of the $\mathrm{z}$-score, is a quantitative measure of confidence (defined as the cumulative $P$-value of molecules in a specific pathway). This measure of confidence, defined on a color scale, indicates the propensity of all the molecules within that pathway to move in a certain direction, toward either increasing or decreasing the likelihood of developing the listed pathology or condition.

\section{Quantitative PCR}

As described above, RNA was isolated from fetal heart tissue. Using the First-strand cDNA Synthesis kit for miRNA (Origene, Rockville, MD, Catalog \#: HP100042), per manufacturer's instructions, RNA was converted to cDNA. The cDNA was used for differential quantification of mRNA transcripts Fibroblast Growth Factor Receptor 1 (Fgfr1), Interleukin-18 (Il-18), and Transforming Growth Factor Beta Receptor 2 (Tgfbr2). ChIP-qPCR was used to assess the Tgfbr2 promoter loci. As described above, chromatin was immunoprecipitated with H3K4me3. DNA was then probed at multiple locations along the Tgfbr2 promoter region in order to construct a histone peak profile. Primer design for both the mRNA and ChIP-qPCR are provided (Additional file 1: Table S4). MRNA was normalized to Beta-Actin ( $\beta$-Actin), while immunoprecipitated DNA was normalized to its respective input control. 


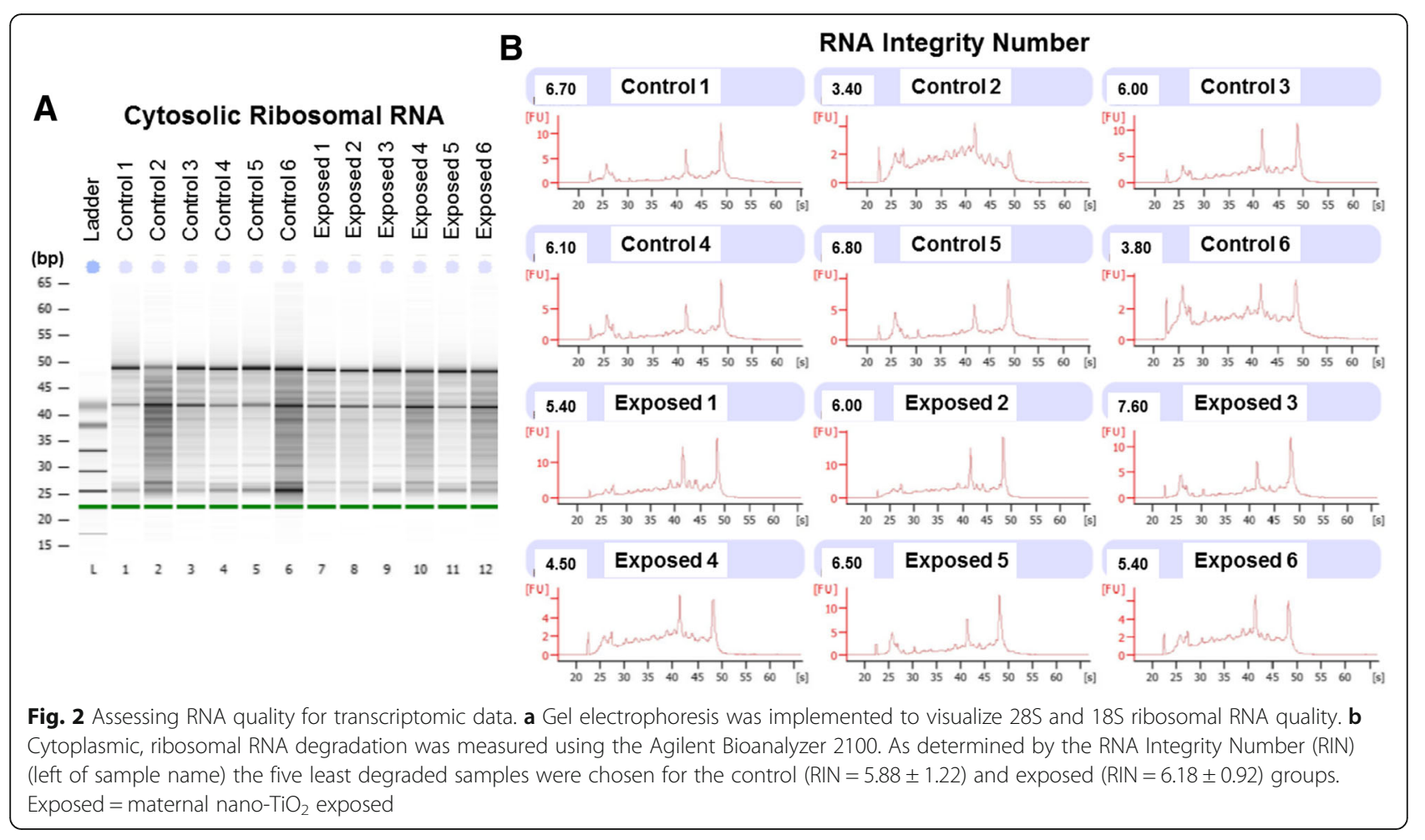

Experiments were performed on the Applied Biosystems 7900HT Fast Real-Time PCR system (Applied Biosystems, Foster City, CA), using 2X SYBR Green Master Mix. Quantification was achieved using the 2- $\Delta \Delta C \mathrm{CT}$ method.

\section{Statistics}

All measures of significance between the control and maternal nano- $\mathrm{TiO}_{2}$ exposure groups for the sequencing data are presented as adjusted $P$-values. Adjusted P-values are a composition of standard, unadjusted P-values and the stringency of the False Discovery Rate (FDR). Differential expression analysis through DESeq2 implements the Wald Test, using multiple testing against the null hypothesis that P-values are uniformly distributed across a data set, known as the Benjamini-Hochberg procedure. The FDR for this study was set at 0.05 . Z-score significance is determined as greater than the absolute value of 2 . The $z$-score is computed as $z=\frac{x}{\sigma_{x}}=\frac{\sum_{i} x_{i}}{\sqrt{n}}=\frac{N_{+}-N_{-}}{\sqrt{N}}$, where $\mathrm{N}_{+}=$the number of molecules following a consistent trend, $\mathrm{N}_{-}=$ the number of molecules following an inconsistent trend, and $\mathrm{N}=$ the number of interactions within a given pathway. In this way, the z-score, using only values with a significant change $(P \leq 0.05)$ can infer direction of a specific pathway while accounting for relationship and data bias and properly weighting the statistical findings (https:// www.qiagenbioinformatics.com/products/ingenuity-pathway-analysis/). A consistency score is the non-statistical assignment of confidence to a specific pathway. Where appropriate, a Student's t-test was used with all data presented as \pm the standard error mean (SEM). Significance is determined as $P \leq 0.05$.

\section{Results}

Animal and $\mathrm{Nano}^{-} \mathrm{TiO}_{2}$ aerosol characteristics

Animal number, age, body weight, and exposure conditions are provided (Table 1). Separate, but similar, inhalation exposures were used for the ChIP and RNA sequencing experiments. No statistical differences were noted between nano- $\mathrm{TiO}_{2}$ exposure in Experimental Group 1 (ChiP Seq) and Experimental Group 2 (RNA Seq). No statistical differences were noted in either progeny weight or total number of pups between maternal nano- $\mathrm{TiO}_{2}$ exposed or control groups.

Representative nano- $\mathrm{TiO}_{2}$ aerosol characterization data are presented in Fig. 3. The target particle concentration was $10 \mathrm{mg} / \mathrm{m}^{3}$ (Fig. 3a). The real-time nano- $\mathrm{TiO}_{2}$ mobility diameter was $129 \mathrm{~nm}$ (Fig. 3b), and the aerodynamic diameter was $143 \mathrm{~nm}$ (Fig. 3c). Nanoparticles were collected on filters, and a representative transmission electron microscopy image is presented in Fig. 3d.

\section{ChIP sequencing ChIP sample metrics}

To better understand the quality and sample dispersion within our cohort for the ChIP sequencing experiment, a series of statistical models were used. To assess the 
Table 1 Animal characteristics

\begin{tabular}{llllllllll}
\hline Exposure Group & Technique & $\begin{array}{l}\text { Number of } \\
\text { animals } \\
(\mathrm{N})\end{array}$ & $\begin{array}{l}\text { Age } \\
\text { (days) }\end{array}$ & $\begin{array}{l}\text { Body } \\
\text { Weight } \\
\text { (grams) }\end{array}$ & $\begin{array}{l}\text { Litter } \\
\text { Size }\end{array}$ & $\begin{array}{l}\text { Pup } \\
\text { Weight } \\
\text { (grams) }\end{array}$ & $\begin{array}{l}\text { Aerosol } \\
\text { Concentration } \\
\left(\mathrm{mg} / \mathrm{m}^{3}\right)\end{array}$ & $\begin{array}{l}\text { Electrical Low- } \\
\text { Pressure Impactor } \\
(\mathrm{nm})\end{array}$ & $\begin{array}{l}\text { Scanning Particle } \\
\text { Mobility Sizer } \\
(\mathrm{nm})\end{array}$ \\
\hline Sham Control & RNA Sequencing & 7 & $109 \pm 7$ & $402 \pm 8.84$ & $13 \pm 2$ & $4.06 \pm 0.16$ & 0 & 0 & 0 \\
Nano- $\mathrm{TiO}_{2}$ & RNA Sequencing & 4 & $113 \pm 2$ & $422 \pm 13.34$ & $14 \pm 1$ & $3.99 \pm 0.22$ & $10.35 \pm 0.13$ & $136.80 \pm 1.44$ & $134.80 \pm 1.24$ \\
Sham Control & ChIP Sequencing & 5 & $104 \pm 2$ & $407 \pm 8.09$ & $12 \pm 2$ & $5.19 \pm 1.02$ & 0 & 0 & 0 \\
Nano- $\mathrm{TiO}_{2}$ & ChIP Sequencing & 6 & $98 \pm 1$ & $376 \pm 19.99$ & $9 \pm 5$ & $4.88 \pm 1.53$ & $10.5 \pm 0.05$ & $143.75 \pm 2.32$ & $129.43 \pm 3.21$ \\
\hline
\end{tabular}

distribution of subpeaks present within the forward and reverse strands of the H3K4me3 and H3K27me3 immunoprecipitations, the average fragment length was determined for each event using the $\mathrm{R}$ package csaw [41]. The cross-correlation graph measures the delay distance, or number of base pairs, which separate distinctive subpeaks, also evaluating the consistency of fragment lengths within the data set (Fig. $4 a$ and b). Multidimensional scaling (MDS) plots were used to evaluate individual library homology between both the H3K4me3 and H3K27me3 groups with the R package edgeR [42]. Log fold change (LogFC) determined the differences between libraries (control, red and maternal nano- $\mathrm{TiO}_{2}$ exposed, blue) within the MDS plots (Fig. 4c and d). To visualize read coverage, the $\mathrm{R}$ packages ChIPpeakAnno and Gviz were installed [43]. Complex, differential binding was assessed for both the H3K4me3 (Fig. 4e) and H3K27me3 (Fig. 4f) binding loci. Together, these results suggest that the immunoprecipitation and chromatin fragmentation were successful, and that differential binding is observed between groups.

\section{ChIP IPA Protein Ontology}

Differential Binding data for both the H3K4me3 and H3K27me3 marks were uploaded and analyzed in QIAGEN's IPA; all changes are shown as maternal nano$\mathrm{TiO}_{2}$ exposed condition relative to the control. Diseases and biological functions $(z$-score $\geq 2)$ for H3K4me3 and H3K27me3 are provided in Additional file 1: Table S1 and $\mathrm{S} 2$, respectively. Of the diseases and biological functions listed, one of the most prominent pathways for H3K4me3 involved infectious disease (Fig. 5a). The heat

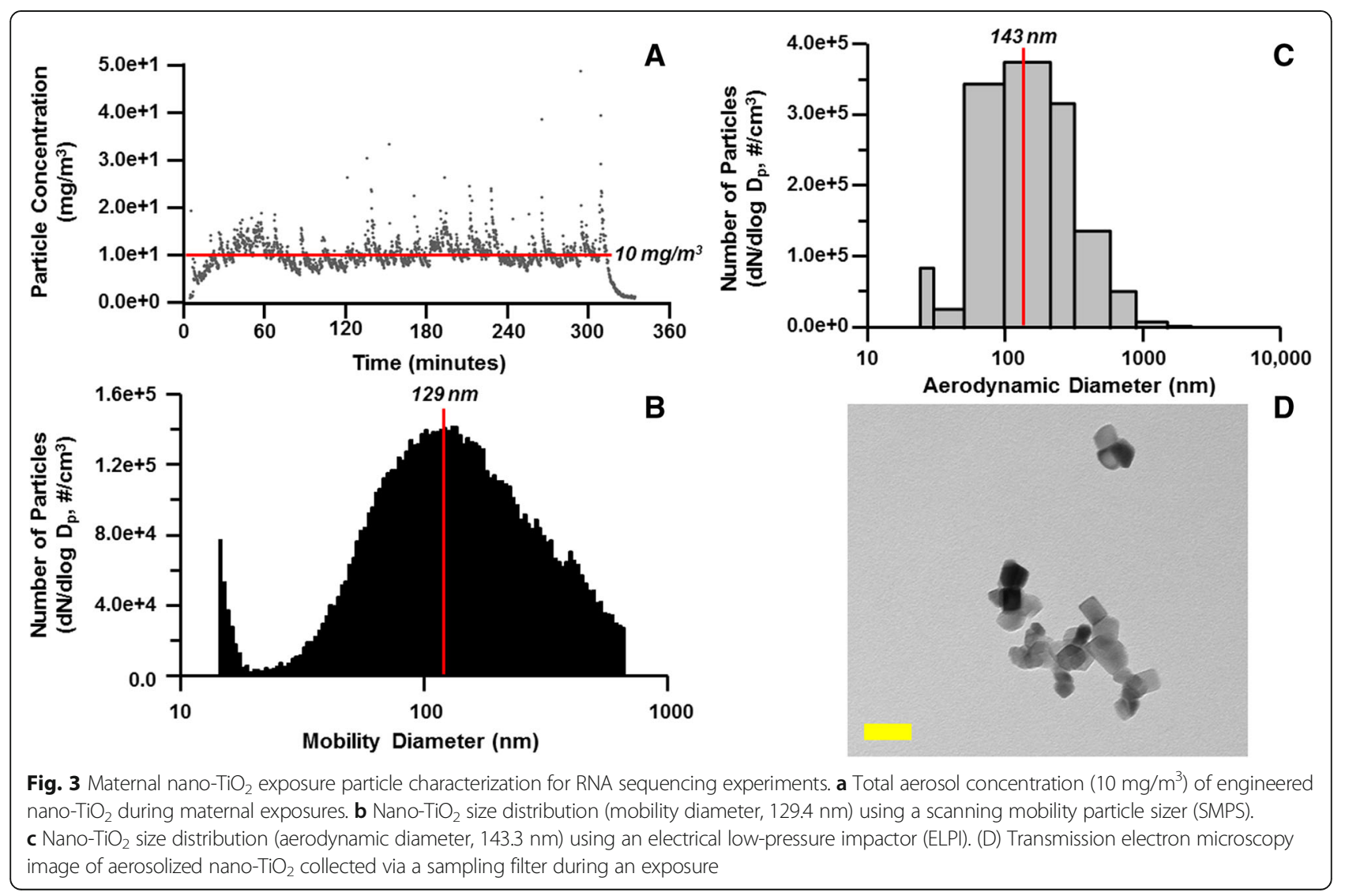




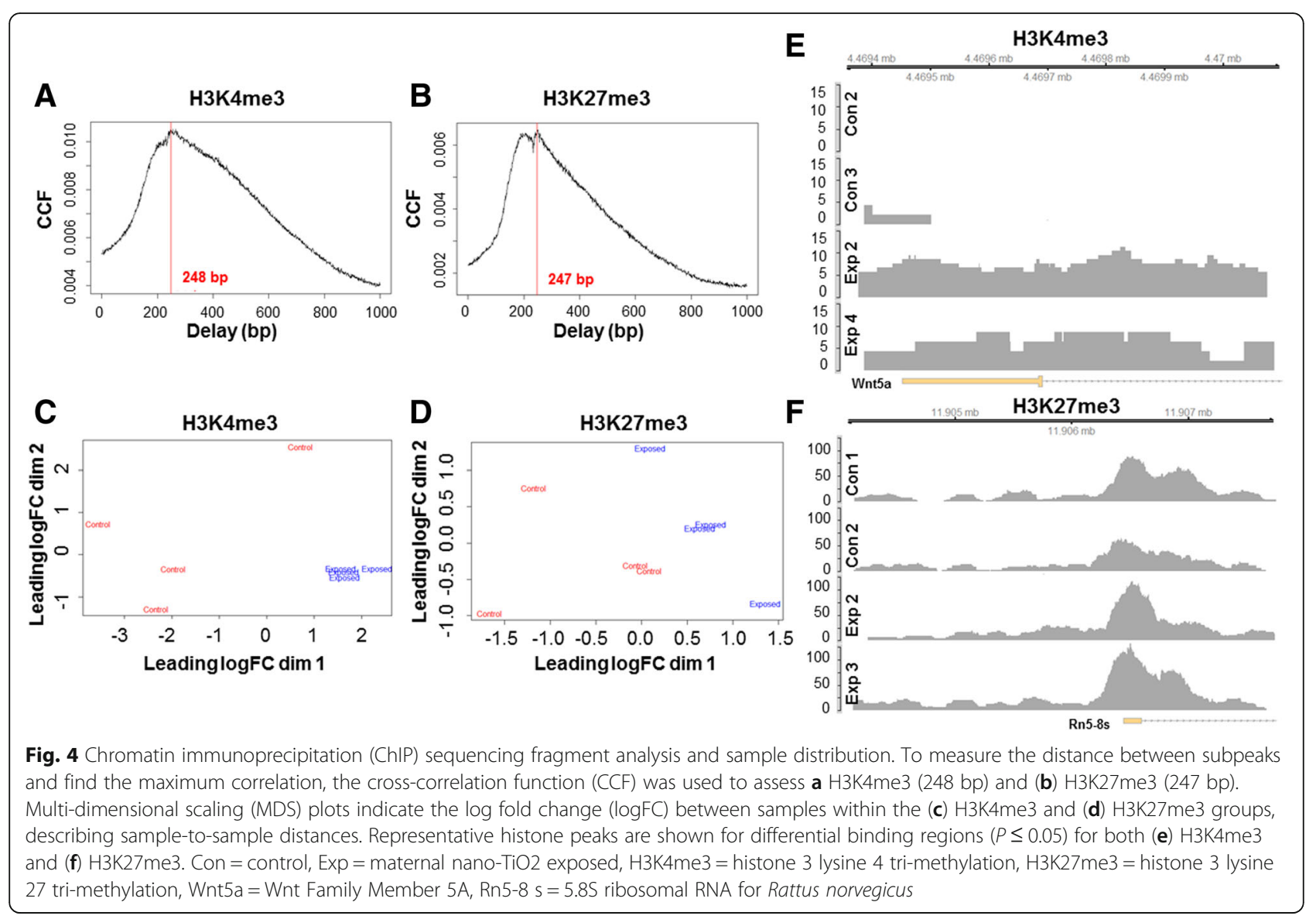

map reveals how changes in molecular signaling could provide an increase susceptibility to infection in maternal nano- $\mathrm{TiO}_{2}$ exposed offspring. The top canonical pathways (z-score $\geq 2$ ) altered during maternal nano$\mathrm{TiO}_{2}$ exposure are presented (Fig. 5b). In general, the canonical pathways altered after exposure involve regulation of growth and cell cycle/apoptosis signaling.

For H3K27me3, the top 10 canonical pathways which are altered are provided (Fig. 5c). For the promoter regions associated with H3K27me3, the majority of signaling changes involve cancer and immunity. A heat map for the toxicological functions of the data representing H3K4me3 is also presented (Fig. 5d). The size and distribution of each major category is proportional to the $\mathrm{z}$-score, which revealed three major organs affected: the heart, kidney and liver. Toxicological pathways associated with the heart, including congenital heart anomaly, heart failure, cardiac hypertrophy (not shown), and cardiac dysfunction (not shown), were found to be significantly decreased in the maternal nano- $\mathrm{TiO}_{2}$ exposed group. Conversely, toxicological pathways associated with the liver and kidney including, renal necrosis and cell death, liver necrosis and cell death, renal damage, and liver damage (not shown) were found to be increased. Also, an increase in red blood cells, and subsequently the hematocrit, were observed.
Increases in H3K4me3 at promoter regions for infection capacity and growth signaling as well as loci involving kidney and liver dysfunction, suggests epigenetic regulation which could significantly alter an organism's susceptibility to disease and potential pre-disposition to future insult. The lack of changes shown for H3K27me3 may suggest an alternative repressive mark implemented as the bivalent companion of H3K4me3.

\section{RNA sequencing RNA sample metrics}

The raw and normalized counts from the RNA sequencing experiment were subjected to a variety of statistical modelling, using the DESeq2 package in R [44], in order to better understand sample parameters. To visualize the variance of the normalized counts data means, the rlog function was used (Fig. 6a). For low-count genes, transformation using rlog, a $\log 2$ scale which normalizes data in reference to the library size, helps to better visualize variance-means. Fig. 6a shows limited outliers within the data set for the control vs. control, but increasing variance in the control vs. maternal nano- $\mathrm{TiO}_{2}$ exposed. Sample-to-sample distance was measured using the PoiClaClu package in R. Sample dissimilarity is depicted as a heat map (Fig. 6b), calculated from the original, not 


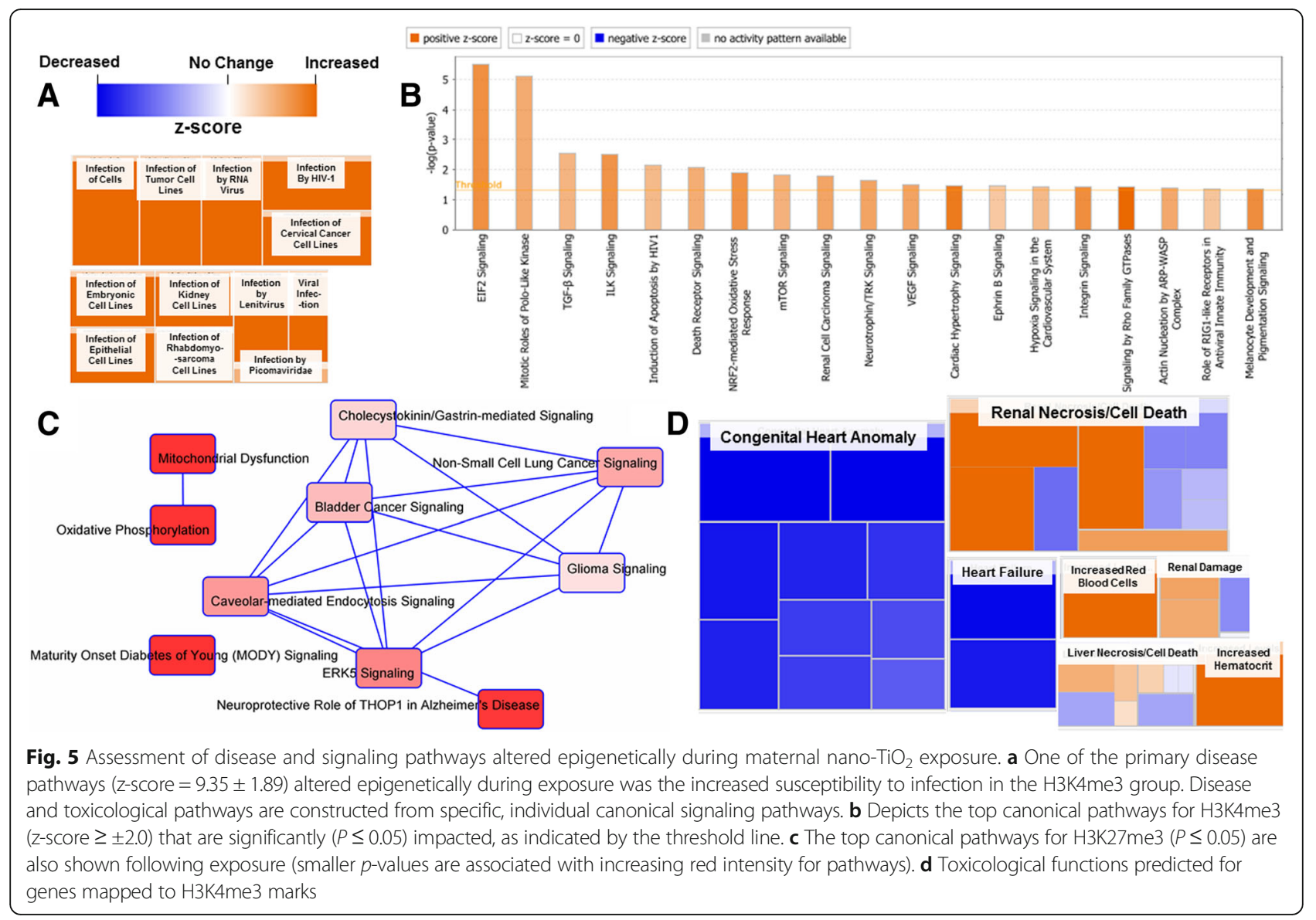

normalized count data. The heat map shows general dissimilarity between the maternal nano- $\mathrm{TiO}_{2}$ exposed and control groups, with the exception of one of the control samples. Another measure implemented for determining sample distance was a multi-dimensional scaling (MDS) plot based on the rlog-normalized counts (Fig. 6c). Again, the plot shows a general dissimilarity between the maternal nano- $\mathrm{TiO}_{2}$ exposed and control cohorts. After performing differential expression analysis with DESeq2, we examined the gene with the lowest associated $p$-value (Fig. 6d). The plot illustrates the similar expression of the gene within each group, while showing the disparities across groups. In Fig. 6e, a MA-plot is used to illustrate the number of genes (red) that fall below the $P$ value of 0.05 . The statistical models used to assess the RNA sequencing samples indicate that normalized count values between groups are similar and that sample homology is close within groups, but not across groups.

\section{RNA IPA protein ontology}

After differential expression analysis processing in $\mathrm{R}$, data was uploaded and analyzed in QIAGEN's IPA; all changes are shown as maternal nano- $\mathrm{TiO}_{2}$ exposed condition relative to the control. Diseases and biological functions ( $\mathrm{z}$ - score $\geq 2$ ) for the RNA are provided in Additional file 1: Table S3. Again, a prominent pathway that was found to be increased in the maternal nano- $\mathrm{TiO}_{2}$ exposed animals involved infectious diseases (Fig. 7a). Both the open promoter conformation (H3K4me3) and the RNA transcript expression reveal an increased propensity for infection. The top canonical pathways ( $\mathrm{z}$-score $\geq 3.45$ ) altered during maternal nano- $\mathrm{TiO}_{2}$ exposure are presented (Fig. $7 \mathrm{~b}$ ). The canonical pathways altered primarily involve inflammatory signaling and organismal development. Examining what factors could be causing differential regulation after maternal nano- $\mathrm{TiO}_{2}$ exposure, we wanted to evaluate molecular regulator effects. The top molecule (consistency score $\geq 10.453$ ) suggested to play a role in differential regulation of pathways was microRNA-145 (Fig. 7c).

In Fig. 7c, it reveals how decreased expression of microRNA-145 can lead to increased expression of pathways involving cell growth and proliferation. A heat map for the toxicological functions of the data representing the RNA is also shown (Fig. 7d). The size and distribution of each major category is proportional to the z-score and, again consistent with the H3K4me3 mark, three major organs were shown to be affected: the heart, kidney and liver. Toxicological pathways associated with the heart, 


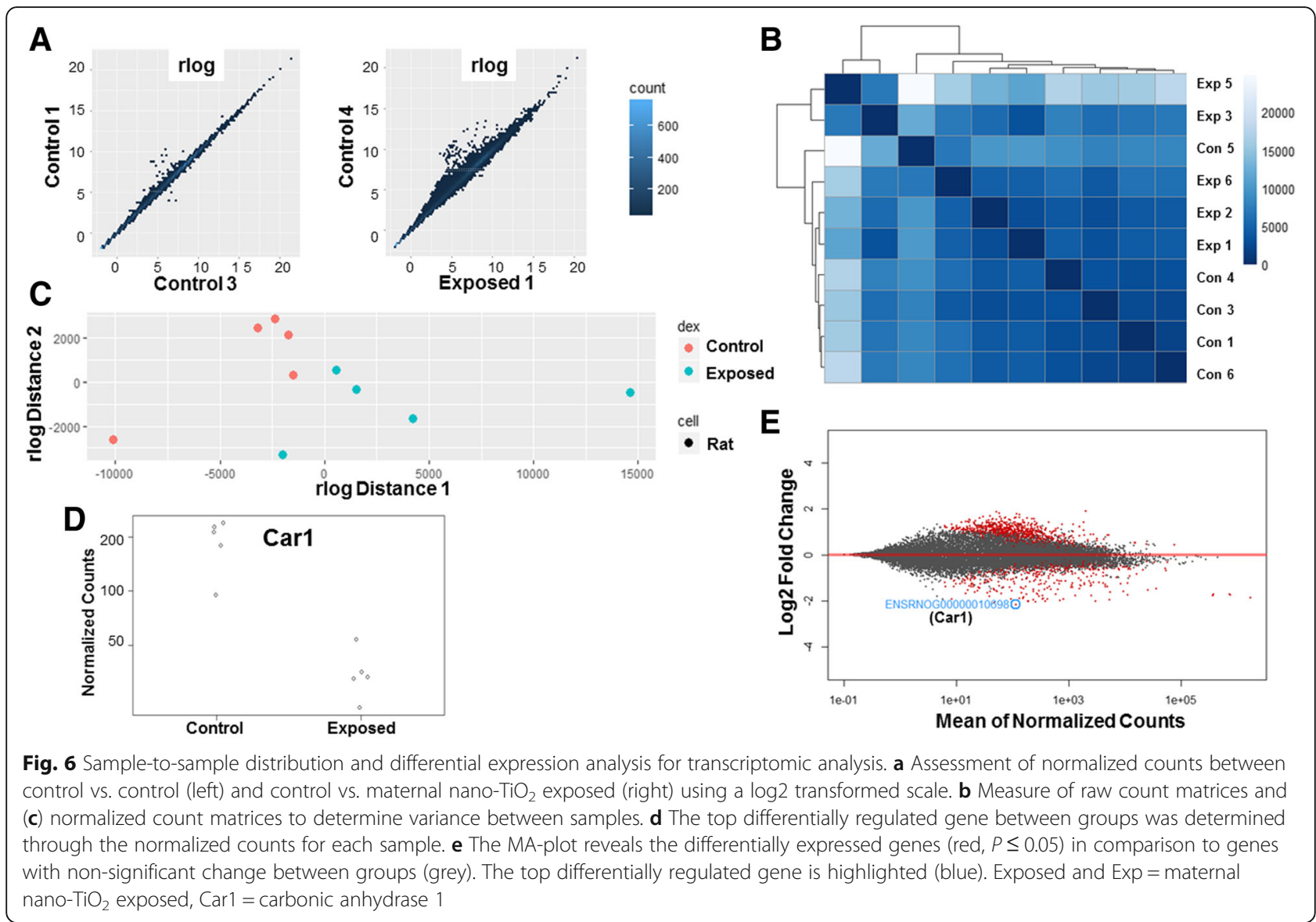

including congenital heart anomaly, cardiac hypoplasia, heart failure, cardiac fibrosis, and cardiac damage, were found to be significantly decreased in the maternal nano$\mathrm{TiO}_{2}$ exposed group. Alternatively, toxicological pathways associated with the liver and kidney including, renal necrosis and cell death, liver hyperplasia/hyperproliferation, renal proliferation, renal damage, and renal autophagy were found to be increased. As reported for the H3K4me3 promoter regions, increased RNA transcription of genes involving red blood production are shown. Similar to the epigenetic modification H3K4me3, the differential expression of transcripts follows a similar pattern of increased infection and growth of the organism, with increased molecular markers of dysfunction in the liver and kidney.

\section{Epigenetic regulation of transcription}

In order to examine how changes between the H3K4me3 mark and RNA transcript data aligned, we performed a comparative analysis through QIAGEN's IPA, all changes are shown as maternal nano-TiO2 exposed condition relative to the control. The top canonical pathways $(\mathrm{z}$-score $\geq 4.5$ ) for both the transcript and ChIP data are shown (Fig. 8a). The combined data sets illustrate the common pathways involving both inflammation and organismal growth signaling. For toxicological functions, the molecular profile for cardiac dysfunction is significantly decreased compared to the controls, while kidney dysfunction is increased (Fig. 8b). A heat map for the cumulative diseases and biological functions is shown (Fig. 8c). The heat map depicts two major molecular changes that could impact the phenotype: increased survival and increased susceptibility to infection. In Fig. 8d, canonical pathways are sorted by $p$ value, depicting pathways with large sets of molecules having significantly altered expression levels. Although, the mitochondrial dysfunction and oxidative phosphorylation pathways do not have significant $\mathrm{z}$-scores and a very small contribution of changes coming from the transcript data, Fig. 8b demonstrates the epigenetic changes occurring at these loci to a large segment of genes. Figure 8e illustrates the NF- $\mathrm{kB}$ (Nuclear Factor kappa-light-chain-enhancer of activated B cells) signaling pathway for the RNA (right) and H3K4me3 (left) sequencing experiments. The comparative analysis suggests that maternal nano- $\mathrm{TiO}_{2}$ exposure can cause significant changes to how the development of the progeny takes place, changing the epigenetic landscape, which can directly affect transcript abundance. 


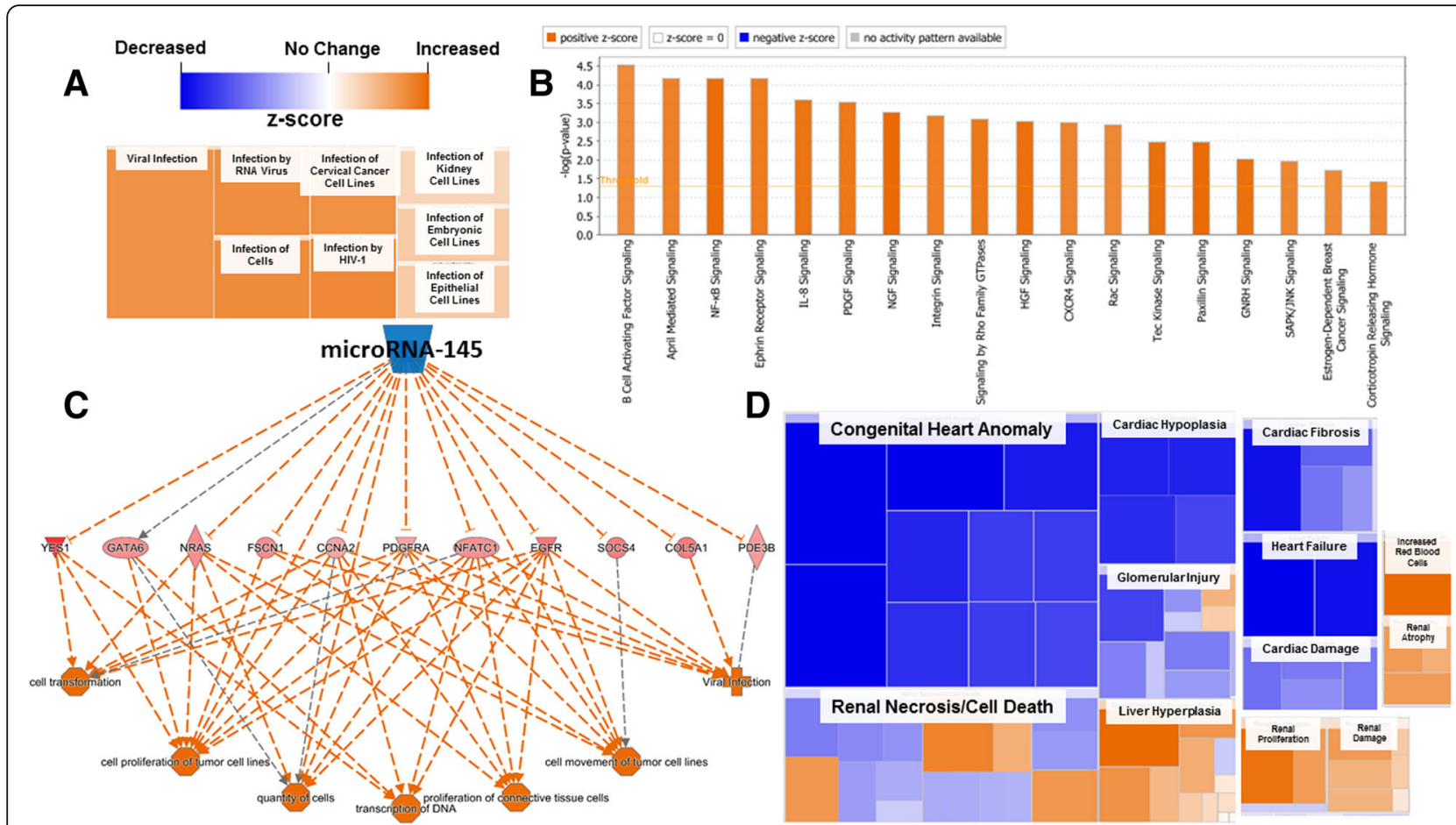

Fig. 7 Assessment of disease and signaling pathways altered transcriptionally during maternal nano- $\mathrm{TiO}_{2}$ exposure. a Similar to the activation by H3K4me3, transcriptional upregulation of genes associated with increased susceptibility to infection $(z-s c o r e=2.02 \pm 0.96)$ was found. $\mathbf{b}$ The top canonical pathways ( $z$-score $\geq \pm 3.45$ ) that are significantly $(P \leq 0.05)$ impacted transcriptionally, as indicated by the threshold line. The canonical pathways for the RNA sequencing reveal a significant increase in inflammatory and growth signaling. $\mathbf{c}$ The top regulator (consistency score $=10.453$ ) determined through pathway analysis of gene expression (arrows = activation, bars = repression). Increasing gene activation (red) and suppression (blue) reveal targeting of multiple cell functions. $\mathbf{d}$ Toxicological functions predicted for transcript abundance in the RNA sequencing experiment

\section{Molecular validation of sequencing}

To further confirm the reliability of the sequencing data, we implemented qPCR to examine molecules involved in the NF- $\mathrm{kB}$ Pathway, which are not shown in the illustrative Fig. 8d, e. The mRNA levels of Fgfr1, Il-18, and Tgfbr2 are reported, and coincide with similar expression profiles seen in the sequencing data (Fig. 9a). In Fig. 9a, the data obtained from RNA sequencing (grey bars) are used as a reference to validate the expression profile of the maternal nano-TiO2 group when running qPCR. Likewise, we also wanted to use ChIP-qPCR to validate that histone modifications were also reliably reported, with the ChIP-Seq revealing epigenetic changes at the Tgfbr2 promoter region. We confirmed the H3K4me3 histone modifications for Tgfbr2, showing higher H3K4me3 association at its promoter region (Fig. 9b). The increased magnitude of the histone peak of the maternal nano-TiO2 group, Fig. 9b, suggests the increased abundance of H3K4me3 and active transcription of the Tgfbr2 gene. Tgfbr2 provides an explicit example of how genes reported to be epigenetically altered (ChIP-Seq, through H3K4me3 localization at the Tgfbr2 promoter region) with subsequent changes in transcription (RNA-Seq, reporting increased expression of Tgfbr2 transcripts) can be further validated using other molecular techniques, such as qPCR. An overview of the experimental design is illustrated in Fig. 9c. Briefly, the figure provides an example of suggested functional outcomes related to maternal nano-TiO2 exposure, with the link between the exposure paradigm and end function being fetal, epigenetic consequences.

\section{Discussion}

The gene expression and epigenetic analyses performed in this study provide the first evidence that maternal ENM inhalation may result in significant pathway alterations in the fetus. The two most prominently impacted mechanisms are: inflammatory signaling, and cardiacrenal-hepatic pathology/toxicity.

The nano- $\mathrm{TiO}_{2}$ exposure paradigm used herein $\left(10 \mathrm{mg} / \mathrm{m}^{3}, 4-6 \mathrm{~h}\right)$ resulted in a calculated lung deposition of approximately $217 \mu \mathrm{g}$. This lung burden, achieved over 7 days of exposure in the second half of gestation, has been previously shown to impair uterine arteriolar reactivity by almost 50\% [40]. To estimate how this lung burden compares to what a human may experience, alveolar surface areas must be known [32]. The rat alveolar surface area is $0.4 \mathrm{~m}^{2} /$ lung. Therefore, the rat 


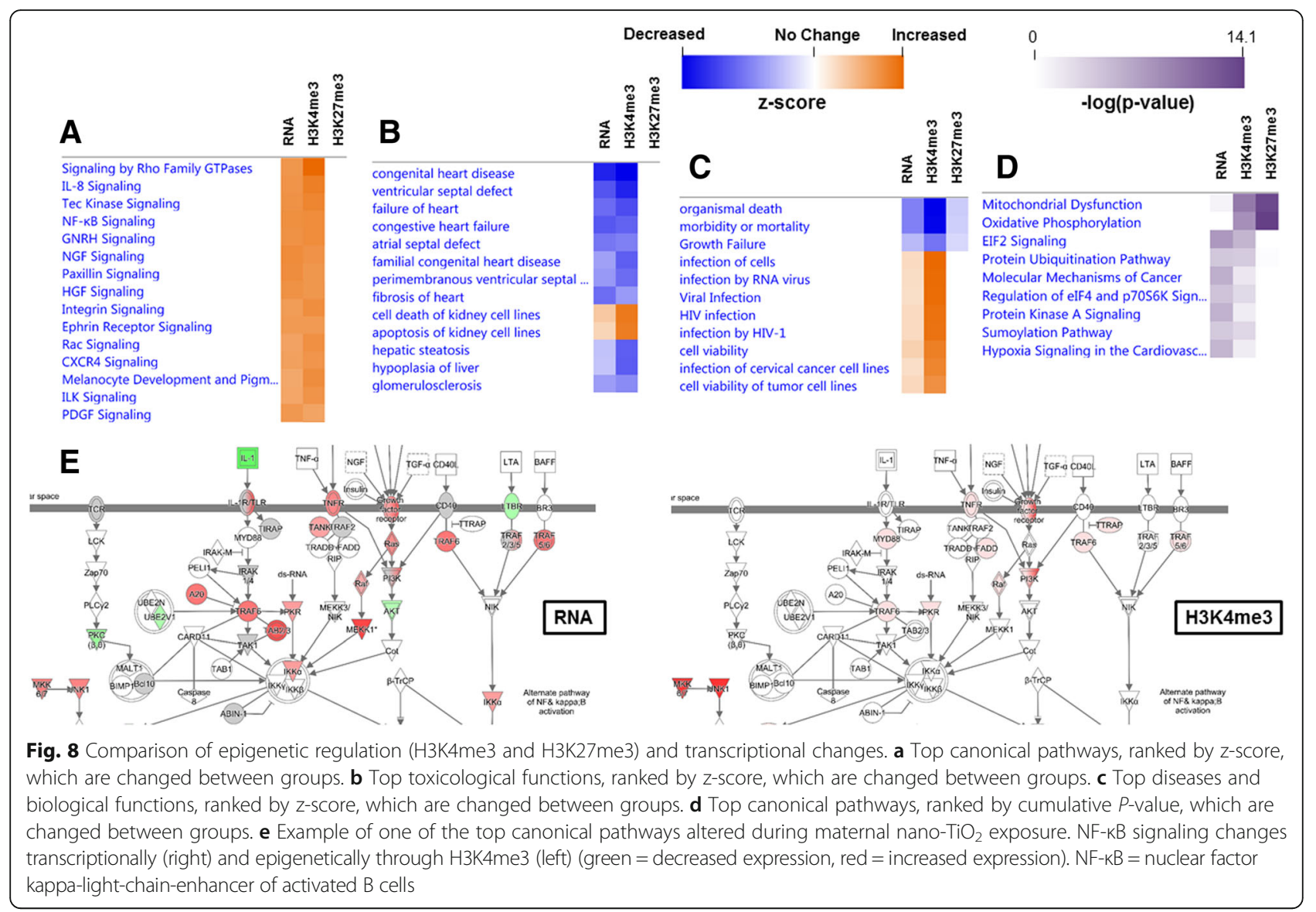

burden of $217 \mu \mathrm{g} / \mathrm{lung}$ would result in $542.5 \mu \mathrm{g} / \mathrm{m}^{2}$. Given that the human alveolar surface area is $102 \mathrm{~m}^{2}$, the equivalent human burden of this exposure paradigm would be $55.3 \mathrm{mg}$. The next logical question is how long would it take to achieve this burden in humans. In this regard, lung burden may be calculated as:

\section{nano-TiO ${ }_{2}$ aerosol concentration $\cdot$ minute ventilation -exposure duration - deposition fraction,}

with the following values:

$$
\begin{aligned}
55.3 \mathrm{mg} & =\text { nano }-\mathrm{TiO}_{2} \text { aerosol concentration } \\
& .7600 \mathrm{ml} / \mathrm{min} \cdot(8 \mathrm{hr} / \text { day } \cdot 60 \mathrm{~min} / \mathrm{hr}) \\
& \cdot 14 \%,
\end{aligned}
$$

and therefore:

$$
\begin{gathered}
55.3 \mathrm{mg}=\text { nano- }-\mathrm{TiO}_{2} \text { aerosol concentration } \\
\cdot 0.51 \mathrm{~m}^{3} / \text { day. }
\end{gathered}
$$

The National Institute for Occupational Safety and Health (NIOSH) Recommended Exposure Limit (REL), or aerosol concentration for nano- $\mathrm{TiO}_{2}$ is $0.3 \mathrm{mg} / \mathrm{m}^{3}$ (DHHS, 2011). This would result in a lung burden of $0.15 \mathrm{mg} /$ day. Whereas, the Occupational Safety and Health Administration (OSHA) Permissible Exposure
Limit is $5 \mathrm{mg} / \mathrm{m}^{3}$ (DHHS 2011). This would result in a lung burden of $2.55 \mathrm{mg} /$ day. Considering the NIOSH REL and OSHA PEL together, it would require 1.45 working years or 21.7 working days (respectively) for a human to achieve comparable lung burdens with the exposure paradigm used herein. Because the human gestational period is 9 months, we consider our exposure paradigm highly relevant to the worker population.

Contrary to the functional deficits seen in the young adult $[20,25]$ we found that both the transcriptomic and epigenetic data support increased cardiac function (Figs. 5d and 7d). Though this seems paradoxical, we suggest that the interplay between the heart, liver, and kidneys is vital in understanding the pathology associated with maternal nano- $\mathrm{TiO}_{2}$ exposure. It is equally plausible that as hematocrit increases, viscosity of the blood also increases, requiring an elevation in contractile force or a drop in peripheral resistance. Alternatively, it is possible that disruptions in maternal-fetal perfusion balances occur. The pulmonary exposure of the mother is well described, but the secondary effect(s) on the developing progeny is/are likely to come through impacts on the maternal/fetal circulation. Maternal nutrients are delivered to the placenta via the arterial circuit, if blood flow is inadequate, then fetal compensation must occur 


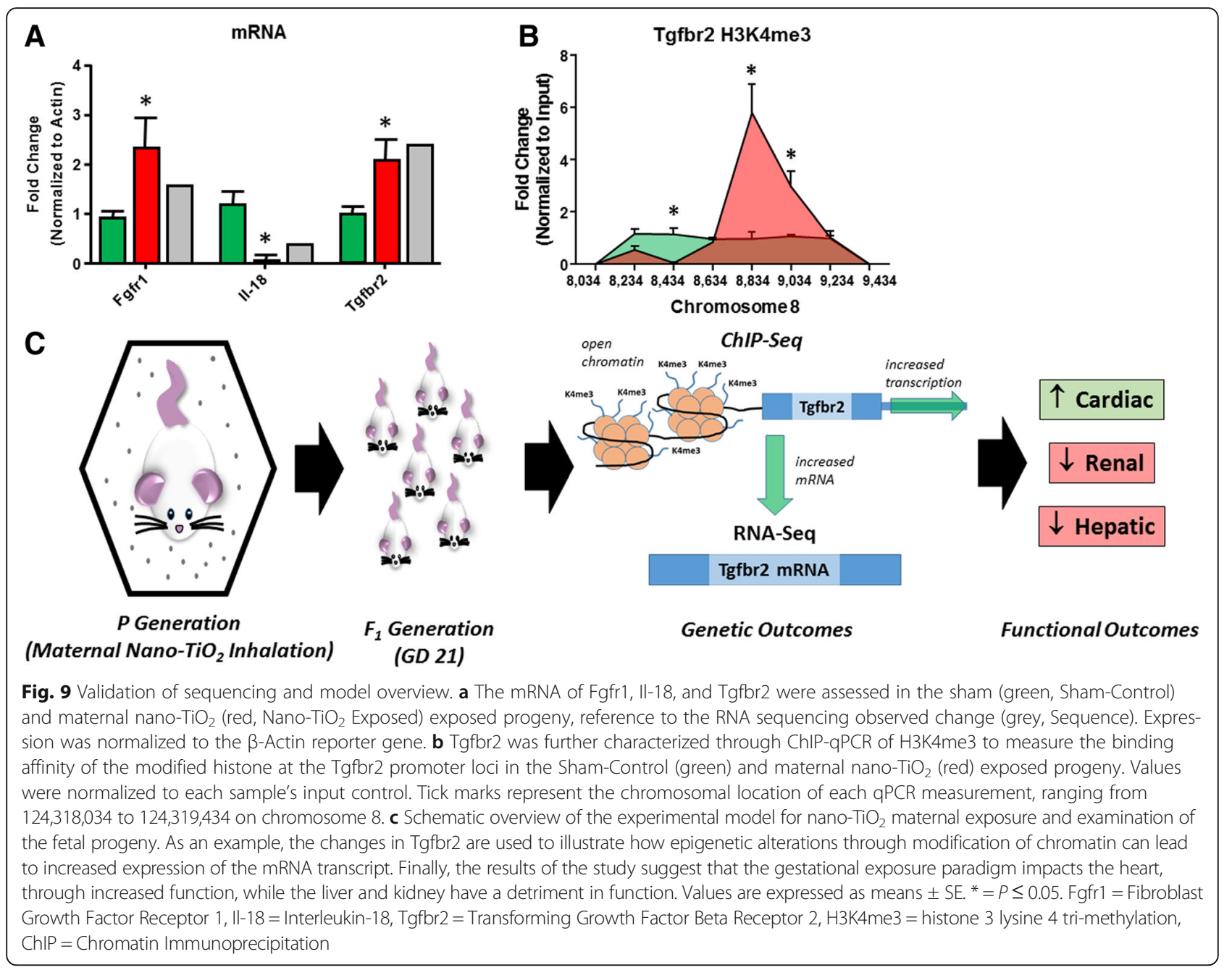

to support proper nutrient delivery via the umbilical vein to the fetal portal circulation.

At the fetal stage, the heart plays a less significant role in energetics [45]. Whereas, the liver and kidneys play pivotal roles in blood conditioning at this stage of development, and these signaling pathways are altered by maternal ENM inhalation during gestation (Fig. 5). We hypothesize that potential liver and kidney damage from either inflammation, direct ENM translocation or a combination may result in an increased hematocrit, and or maternal-fetal perfusion balance. Together, this may suggest that in maternal nano- $\mathrm{TiO}_{2}$ exposed progeny, the functional deficits seen later in development may be a result of this initial hepatic and renal insult, with subsequent cardiac overcompensation which may represent a protective mechanism. These findings correspond to reports of hepatic DNA damage in newborn murine offspring after maternal nano- $\mathrm{TiO}_{2}$ inhalation [46]. Impairments in renal function may have profound effects on tubuloglomerular feedback, the renin angiotensin system, and/or osmotic regulation. These impairments may collectively or individually directly influence cardiovascular health throughout prenatal and postnatal development.

MicroRNA (miRNA) are well known to be altered by transcriptomic and epigenetic regulators. When expressed, miRNA broadly regulate cellular function [47] and have been implicated in numerous epigenetic pathways [48]. In Fig. 7c transcriptomic data is provided that reflects the most consistently altered regulator after maternal nano- $\mathrm{TiO}_{2}$ inhalation. Decreased expression of miRNA-145 has been suggested to increase protein synthesis of targets directly involved in signaling events that promote organism growth and development. The role of altered miRNAs in progeny after maternal ENM inhalation is poorly understood, and may provide a better understanding of the relationship between ENM toxicities, epigenetics, and gene expression.

Figure $8 \mathrm{c}$ presents an overview of the two primary cell signaling pathways that are altered during gestational exposure: immunity and development. Parameters of organismal health and development are presented largely as molecular markers for cardiac signaling and function. The increased gene expression of molecular markers 
associated with infection and immunity may indicate the likelihood of autoimmune disorders associated with an overactive immune system. This is most evident when considering the inflammatory pathways indicated in Fig. 8a and the target organ (kidney) indicated in Fig. 8b reflected by an increased susceptibility as shown in Fig. 8c. These molecular markers may also represent the consequence presented in Fig. 8a of a proinflammatory environment; such an environment has been associated with chronic conditions including cardiovascular disease and cancer [49]. Pulmonary exposure to carbon black nanoparticles has also been identified to contribute to the development of immunotoxicity, particularly in lymphoid organs [22]. Interestingly, organismal death and morbidity/mortality appears to be decreased in maternal nano- $\mathrm{TiO}_{2}$ offspring, which may again seem counterintuitive. However, we speculate this may reflect a greater systemic response to compensate for the numerous other mechanisms disturbed by ENM inhalation during gestation.

To better identify the future consequences of ENM exposure, the significance of the pathways was represented as the change in $P$-value (Fig. 8d). Mitochondrial dysfunction and oxidative phosphorylation appeared to have the greatest changes in methylation, indicating that future complications in these pathways may occur. Given their widespread involvement, this epigenetic predisposition may manifest in any tissue. In other words, the epigenetic changes associated with energetics may reflect significant alterations that occur during fetal development. It is important to indicate that these changes may not be manifested in functional transcriptomic or proteomic changes until postnatal development or even later into adulthood. If correct, this would be consistent with the Barker Hypothesis and $\mathrm{DOHaD}$.

Maternal nano- $\mathrm{TiO}_{2}$ exposure is also associated with a pronounced effect on key inflammatory pathways in the exposed progeny. In Fig. 8e, protein kinase B (AKT) signaling is decreased, potentially resulting in an impairment in calcium-independent nitric oxide signaling which would likely result in dysfunctional endothelium-dependent responses. Indeed, calcium dependent and independent mechanisms, as well as endothelial arteriolar dilation are significantly impaired at 3-4 weeks of age [50]. Furthermore, augmented NF- $\mathrm{kB}$ signaling via both alternate and canonical pathways [51] has been reported. Maternal nano- $\mathrm{TiO}_{2}$ exposure significantly activated the expression of the Lymphotoxin Beta Receptor (LTBR) gene, while suppressing the expression of the regulating enzyme Inhibitor of NF- $\kappa B$ Kinase Subunit Alpha (IKK $\alpha$ ) Fig. 8e. This is important in the negative feedback of the NF-kB canonical signaling that limits inflammatory gene activation and suggests that more robust inflammatory responses are possible as evidenced in Fig. 8a. Furthermore,
NF- $\mathrm{KB}$ plays a central role in the development of inflammation through further regulation of genes encoding not only pro-inflammatory cytokines, but also adhesion molecules such as E-selectin, VCAM-1 (vascular cell adhesion molecule-1) and ICAM-1 (intercellular adhesion molecule1), chemokines, and inducible nitric oxide synthase (iNOS) $[52,53]$. Figure $8 \mathrm{e}$ also reflects a significant increase in interleukin-8 (IL-8) signaling, a major chemokine associated with neutrophil chemotaxis and degranulation secreted by macrophages and endothelial cells during acute inflammatory responses [54]. Considered jointly, uncontrolled activation of NF- $\mathrm{kB}$ and IL-8 pathways in maternally exposed progeny may predispose towards endothelialdependent dysfunction and leukocyte adhesion.

\section{Conclusion}

The pathway analyses reported herein indicate dysfunction in many physiologic systems. As it is not possible to functionally verify each of these functional implications, the primary goal of the manuscript is to identify those systems as a priority for future study. Systemic impairments associated with acute and chronic nanomaterial exposures is an evolving field as nanotechnology continues to expand. Maternal and fetal outcomes following gestational exposures have recently been considered. While initial functional microvascular assessments have begun, little is known regarding epigenetic alterations within the F1 generation. The findings from this study describe epigenetic changes in the progeny of mothers exposed to nano- $\mathrm{TiO}_{2}$ aerosols during gestation. The evidence of the study is strengthened by the use of two separate cohorts to separately probe the transcriptomic and epigenetic alterations, suggesting that even in separate discrete experimental populations, changes to the epigenome and RNA transcript levels align and similar exposure paradigms yield consistent results. Changes in the RNA transcripts and histone modifications on DNA suggest that maternal nano- $\mathrm{TiO}_{2}$ progeny exhibit a propensity toward hepatic and renal disease, increased inflammatory signaling, and growth/survival while showing decreased cardiac dysfunction. What remains to be understood is if and/or how far these epigenetic changes persistent into adulthood, the dose-response relationships, and what stage of development is most sensitive to maternal ENM exposure.

\section{Additional file}

Additional file 1: Table S1. Listing of all disease and biological pathways with a positive or negative $z$-score greater than the absolute value of 2 for H3K4me3. Table S2. Listing of all disease and biological pathways with a positive or negative $z$-score greater than the absolute value of 2 for H3K27me3. Table S3. Listing of all disease and biological pathways with a positive or negative $z$-score greater than the absolute value of 2 for RNA. Table S4. Primer design for qPCR experiments. 
Primers for the mRNA-qPCR experiments were designed using Primer-BLAST (https:/www.ncbi.nlm.nih.gov/tools/primer-blast/). MRNA primers were designed to produced amplicons $100-250 \mathrm{bp}$ in length. ChIP-qPCR primers were designed spanning a $1400 \mathrm{bp}$ loci in the promoter region of the Tgfbr2 gene. Primers were designed to measure chromatin H3K4me3 modifications every $200 \mathrm{bp}$. Primer sequences were constrained to 60-100 bp amplicon lengths, in order to appropriately span sheered chromatin. All primer designs were performed against the Rattus norvegicus July 2014 (RGSC 6.0/rn6) genome build. ChIP = Chromatin Immunoprecipitation. (DOCX 41 kb)

\section{Abbreviations}

AKT: Protein Kinase B; Car1: Carbonic anhydrase 1; ChIP: Chromatin Immunoprecipitation; DOHad: Developmental Origin of Health and Disease; ENM: Engineered Nanomaterials; FDR: False Discovery Rate; Fgfr1: Fibroblast Growth Factor Receptor 1; GD: Gestational Day; H3K27me3: 3 lysine 27 trimethylation; H3K4me3: 3 lysine 4 tri-methylation; IACUC: Institutional Animal Care and Use Committee; ICAM-1: Intercellular Adhesion Molecule-1; IKKa: Inhibitor of NF-KB Kinase Subunit Alpha; II-18: Interleukin-18; IL8: Interleukin 8; IPA: Ingenuity Pathway Analysis; LogFC: Log Fold Change; LTBR: Lymphotoxin Beta Receptor; NF-KB: Nuclear Factor kappa-light-chainenhancer of activated B cells; NOS: Nitric Oxide Synthase; PEL: Permissible Exposure Limit; qPCR: Quantitative Polymerase Chain Reaction;

REL: Recommended Exposure Limit; RIN: RNA Integrity Number; Rn5-8 s: $5.8 \mathrm{~S}$ ribosomal RNA for Rattus norvegicus; Tgfbr2: Transforming Growth Factor Beta Receptor 2; VCAM-1: Vascular Cell Adhesion Molecule-1; Wnt5a: Wnt Family Member $5 \mathrm{~A}$

\section{Acknowledgements}

We thank Kevin Engels of the West Virginia University Department of Physiology, Pharmacology and Neuroscience; and Sherri A. Friend of NIOSH for their expert technical assistance.

\section{Funding}

This work was supported by: NIH-R00-ES024783 (PAS), R01-ES015022 (TRN), R01 HL-128485 (JMH), AHA-13PRE16850066 (CEN), NSF-1003907 (TRN), DGE1144676 (QAH, ABA, TRN), and AHA-17PRE33660333 (QAH).

\section{Availability of data and materials}

The datasets supporting the conclusions of this article are included within the article and its Additional file.

\section{Authors' contributions}

PAS-study design, pregnancy model, inhalation exposures, tissue harvesting, manuscript preparation. QAH-transcriptomic analysis, manuscript preparation. CEN- transcriptomic analysis, manuscript preparation. ABA- tissue harvesting, manuscript preparation. MVP-transcriptome preparation. DLS-transcriptome preparation. CRM-pregnancy model, inhalation exposures, tissue harvesting. JY-inhalation exposures. JMH-transcriptome analysis design, manuscript preparation. VCC- lung burden calculations, rodent to human comparisons, manuscript preparation. TRN- study design, pregnancy model, inhalation exposures, tissue harvesting, manuscript preparation. All authors read and approved the final manuscript.

\section{Ethics approval and consent to participate}

not applicable

\section{Consent for publication}

The authors consent to publication.

\section{Competing interests}

The authors declare that they have no competing interests.

\section{Publisher's Note}

Springer Nature remains neutral with regard to jurisdictional claims in published maps and institutional affiliations.

\section{Author details}

'Department of Pharmacology and Toxicology, Ernest Mario School of Pharmacy, Rutgers University, Piscataway, NJ, USA. ${ }^{2}$ Environmental and Occupational Health Sciences Institute, Piscataway, NJ, USA. ${ }^{3}$ Division of
Exercise Physiology, West Virginia University School of Medicine, Morgantown, WV, USA. ${ }^{4}$ Mitochondria, Metabolism \& Bioenergetics Working Group, West Virginia University School of Medicine, Morgantown, WV, USA. ${ }^{5}$ Immunity, Inflammation, and Disease Laboratory, National Institute of Environmental Health Sciences, Research Triangle Park, NC, USA. ${ }^{6}$ Toxicology Working Group, West Virginia University School of Medicine, Morgantown, WW, USA. ${ }^{7}$ Department of Pharmaceutical Sciences, West Virginia University School of Pharmacy, Morgantown, USA. ${ }^{8}$ Department of Physiology, Pharmacology, and Neuroscience, Robert C. Byrd Health Sciences Center, West Virginia University School of Medicine, 1 Medical Center Drive, Morgantown, WV 26506-9229, USA.

Received: 4 October 2017 Accepted: 21 December 2017

Published online: 10 January 2018

\section{References}

1. de Boo HA, Harding JE. The developmental origins of adult disease (barker) hypothesis. Aust N Z J Obstet Gynaecol. 2006;46(1):4-14.

2. Heindel JJ, Vandenberg LN. Developmental origins of health and disease: a paradigm for understanding disease cause and prevention. Curr Opin Pediatr. 2015;27(2):248-53.

3. Lane $\mathrm{RH}$. Fetal programming, epigenetics, and adult onset disease. Clin Perinatol. 2014;41(4):815-31.

4. Alexander BT, Dasinger JH, Intapad S. Fetal programming and cardiovascular pathology. Compr Physiol. 2015;5(2):997-1025

5. Grason HA, Misra DP. Reducing exposure to environmental toxicants before birth: moving from risk perception to risk reduction. Public Health Rep. 2009;124(5):629-41.

6. He $\mathrm{M}$, et al. Exposure to bisphenol a enhanced lung eosinophilia in adult male mice. Allergy Asthma Clin Immunol. 2016;12:16.

7. Dadvand $\mathrm{P}$, et al. Maternal exposure to particulate air pollution and term birth weight: a multi-country evaluation of effect and heterogeneity. Environ Health Perspect. 2013;121(3):267-373.

8. Perez-Hernandez $M$, et al. Multiparametric analysis of anti-proliferative and apoptotic effects of gold nanoprisms on mouse and human primary and transformed cells, biodistribution and toxicity in vivo. Part Fibre Toxicol. 2017:14(1):41.

9. Adamcakova-Dodd A, et al. Effects of prenatal inhalation exposure to copper nanoparticles on murine dams and offspring. Part Fibre Toxicol. 2015;12:30.

10. Abukabda AB, Stapleton PA, Nurkiewicz TR. Metal Nanomaterial toxicity variations within the vascular system. Curr Environ Health Rep. 2016; 3(4):379-91.

11. Yan Z, et al. Zinc oxide nanoparticle-induced atherosclerotic alterations in vitro and in vivo. Int J Nanomedicine. 2017;12:4433-42.

12. Holland NA, et al. Impact of pulmonary exposure to gold core silver nanoparticles of different size and capping agents on cardiovascular injury. Part Fibre Toxicol. 2016;13(1):48.

13. Hougaard KS, et al. A perspective on the developmental toxicity of inhaled nanoparticles. Reprod Toxicol. 2015;56:118-40.

14. Jackson $P$, et al. Exposure of pregnant mice to carbon black by intratracheal instillation: toxicogenomic effects in dams and offspring. Mutat Res. 2012; 745(1-2):73-83.

15. Blum $\mathrm{J}$, et al. Effects of maternal exposure to cadmium oxide Nanoparticles during pregnancy on maternal and offspring kidney injury markers using a Murine model. J Toxicol Environ Health A. 2015;78(12):711-24.

16. Campagnolo $L$, et al. Silver nanoparticles inhaled during pregnancy reach and affect the placenta and the foetus. Nanotoxicology. 2017;11(5):687-98.

17. Ema M, et al. Reproductive and developmental toxicity of carbon-based nanomaterials: a literature review. Nanotoxicology. 2016;10(4):391-412.

18. Hougaard KS, et al. Effects of prenatal exposure to surface-coated nanosized titanium dioxide (UV-titan). A study in mice. Part Fibre Toxicol. 2010;7:16.

19. Engler-Chiurazzi $E B$, et al. Impacts of prenatal nanomaterial exposure on male adult Sprague-Dawley rat behavior and cognition. J Toxicol Environ Health A. 2016;79(11):447-52.

20. Hathaway QA, et al. Maternal-engineered nanomaterial exposure disrupts progeny cardiac function and bioenergetics. Am J Physiol Heart Circ Physiol. 2017;312(3):H446-58.

21. Stapleton PA, et al. Maternal engineered nanomaterial exposure and fetal microvascular function: does the barker hypothesis apply? Am J Obstet Gynecol. 2013;209(3):227. e1-11 
22. El-Sayed YS, et al. Carbon black nanoparticle exposure during middle and late fetal development induces immune activation in male offspring mice. Toxicology. 2015;327:53-61.

23. Kyjovska ZO, et al. Daily sperm production: application in studies of prenatal exposure to nanoparticles in mice. Reprod Toxicol. 2013;36:88-97.

24. Hougaard KS, et al. Effects of lung exposure to carbon nanotubes on female fertility and pregnancy. A study in mice. Reprod Toxicol. 2013;41:86-97.

25. Stapleton PA, et al. Microvascular and mitochondrial dysfunction in the female F1 generation after gestational TiO2 nanoparticle exposure. Nanotoxicology. 2015;9(8):941-51.

26. Hu X, et al. Toxicity evaluation of exposure to an atmospheric mixture of polychlorinated biphenyls by nose-only and whole-body inhalation regimens. Environ Sci Technol. 2015;49(19):11875-83.

27. Stapleton PA. Gestational nanomaterial exposures: microvascular implications during pregnancy, fetal development and adulthood. J Physiol. 2016;594(8):2161-73.

28. Knuckles TL, et al. Nanoparticle inhalation alters systemic arteriolar vasoreactivity through sympathetic and cyclooxygenase-mediated pathways. Nanotoxicology. 2012;6(7):724-35.

29. Nurkiewicz TR, et al. Nanoparticle inhalation augments particle-dependent systemic microvascular dysfunction. Part Fibre Toxicol. 2008;5:1.

30. Yi J, et al. Whole-body nanoparticle aerosol inhalation exposures. J Vis Exp. 2013;75:e50263.

31. Sager TM, Kommineni C, Castranova V. Pulmonary response to intratracheal instillation of ultrafine versus fine titanium dioxide: role of particle surface area. Part Fibre Toxicol. 2008;5:17.

32. Nurkiewicz TR, et al. Pulmonary particulate matter and systemic microvascular dysfunction. Res Rep Health Eff Inst. 2011;164:3-48.

33. Stapleton PA, et al. Impairment of coronary arteriolar endotheliumdependent dilation after multi-walled carbon nanotube inhalation: a timecourse study. Int J Mol Sci. 2012;13(11):13781-803.

34. Bolger AM, Lohse M, Usadel B. Trimmomatic: a flexible trimmer for Illumina sequence data. Bioinformatics. 2014;30(15):2114-20.

35. Shen $L$, et al. diffReps: detecting differential chromatin modification sites from ChIP-seq data with biological replicates. PLoS One. 2013;8(6):e65598.

36. Kim D, Langmead B, Salzberg SL. HISAT: a fast spliced aligner with low memory requirements. Nat Methods. 2015;12(4):357-60.

37. Li $\mathrm{H}$, et al. The sequence alignment/map format and SAMtools. Bioinformatics. 2009;25(16):2078-9.

38. Liao Y, Smyth GK, Shi W. The subread aligner: fast, accurate and scalable read mapping by seed-and-vote. Nucleic Acids Res. 2013;41(10):e108.

39. Liao Y, Smyth GK, Shi W. featureCounts: an efficient general purpose program for assigning sequence reads to genomic features. Bioinformatics. 2014;30(7):923-30.

40. Lun AT, Chen Y, Smyth GK. It's DE-licious: a recipe for differential expression analyses of RNA-seq experiments using quasi-likelihood methods in edgeR. Methods Mol Biol. 2016;1418:391-416.

41. Lun AT, Smyth GK. Csaw: a bioconductor package for differentia binding analysis of ChIP-seq data using sliding windows. Nucleic Acids Res. 2016;44(5):e45.

42. Robinson MD, McCarthy DJ, Smyth GK. edgeR: a bioconductor package for differential expression analysis of digital gene expression data. Bioinformatics. 2010;26(1):139-40.

43. Hahne F, Ivanek R. Visualizing genomic data using Gviz and bioconductor. Methods Mol Biol. 2016;1418:335-51.

44. Love MI, Huber W, Anders S. Moderated estimation of fold change and dispersion for RNA-seq data with DESeq2. Genome Biol. 2014;15(12):550.

45. Taegtmeyer $H$, Sen S, Vela D. Return to the fetal gene program: a suggested metabolic link to gene expression in the heart. Ann N Y Acad Sci. 2010;1188:191-8.

46. Jackson $\mathrm{P}$, et al. Pulmonary exposure to carbon black by inhalation or instillation in pregnant mice: effects on liver DNA strand breaks in dams and offspring. Nanotoxicology. 2012;6(5):486-500.

47. Shukla GC, Singh J, Barik S. MicroRNAs: processing, maturation, target recognition and regulatory functions. Mol Cell Pharmacol. 2011;3(3):83-92.

48. Bartel DP. MicroRNAs: target recognition and regulatory functions. Cell. 2009:136(2):215-33.

49. Singh-Manoux A, et al. Association between inflammatory biomarkers and all-cause, cardiovascular and cancer-related mortality. Can Med Assoc J. 2017;189(10):E384-90.
50. Nurkiewicz TR, Boegehold MA. Calcium-independent release of endothelial nitric oxide in the arteriolar network: onset during rapid juvenile growth. Microcirculation. 2004;11(6):453-62.

51. Ze Y, et al. TiO2 nanoparticles induced hippocampal neuroinflammation in mice. PLoS One. 2014;9(3):e92230.

52. Imai $Y$, et al. Interaction between cytokines and inflammatory cells in islet dysfunction, insulin resistance and vascular disease. Diabetes Obes Metab. 2013;15(Suppl 3):117-29.

53. Sprague AH, Khalil RA. Inflammatory cytokines in vascular dysfunction and vascular disease. Biochem Pharmacol. 2009;78(6):539-52.

54. Harada A, et al. Essential involvement of interleukin-8 (IL-8) in acute inflammation. J Leukoc Biol. 1994;56(5):559-64.

\section{Submit your next manuscript to BioMed Central and we will help you at every step:}

- We accept pre-submission inquiries

- Our selector tool helps you to find the most relevant journal

- We provide round the clock customer support

- Convenient online submission

- Thorough peer review

- Inclusion in PubMed and all major indexing services

- Maximum visibility for your research

Submit your manuscript at www.biomedcentral.com/submit
) Biomed Central 